\title{
FACTORES SOCIODEMOGRÁFICOS Y EVENTOS DE VIDA TEMPRANOS ASOCIADOS CON LA FELICIDAD EN ADULTOS DE LIMA METROPOLITANA
}

\author{
Javier E. Saavedra (1) 1,2,a \\ 1 Instituto Nacional de Salud Mental «Honorio Delgado - Hideyo Noguchi», Lima, Perú. \\ 2 Facultad de Medicina, Universidad Peruana Cayetano Heredia, Lima, Perú. \\ a Médico psiquiatra, doctor en Medicina.
}

\section{RESUMEN}

Objetivos: Estimar los factores sociodemográficos y eventos de vida tempranos asociados con la felicidad en adultos de Lima Metropolitana. Materiales y métodos: Se realizó un análisis secundario del Estudio Epidemiológico de Salud Mental de Lima Metropolitana 2012, el cual utilizó los instrumentos de índice de calidad de vida, la versión breve y modificada del EMBU, un cuestionario de eventos adversos tempranos, un índice de felicidad construido a partir del ítem único sobre felicidad de Andrews y la escala de satisfacción con la vida. Se realizaron análisis multivariados ajustados. Resultados: La felicidad se asoció con el sexo, estado civil, pobreza y nivel de instrucción. Los estilos de crianza asociados con menor felicidad fueron: las conductas de rechazo y las actitudes machistas, y con mayor felicidad: el calor emocional. No se encontró asociación con prácticas de crianza de sobreprotección o favoritismo. Como eventos adversos tempranos se asociaron significativamente: discusiones dentro de la familia, padre con problemas de alcohol, ausencia paterna, conductas de amenazas o chantaje emocional. Conclusiones: Los hallazgos resaltan la importancia de desarrollar programas intensivos en los primeros años de vida que estimulen estilos de crianza y ambientes de desarrollo saludables.

Palabras Clave: Felicidad; Adultos Sobrevivientes de Eventos Adversos Infantiles; Crianza del niño (fuente: DeCS BIREME).

\section{SOCIO-DEMOGRAPHIC FACTORS AND EARLY LIFE EVENTS ASSOCIATED WITH HAPPINESS IN ADULTS OF METROPOLITAN LIMA}

\begin{abstract}
Objectives: To estimate socio-demographic factors and early life events associated with happiness in adults in metropolitan Lima. Materials and methods: A secondary analysis was carried out on the Epidemiological Study of Mental Health of Metropolitan Lima 2012. The instruments used were the Quality of Life Index; a brief and modified version of the EMBU; a questionnaire of early adverse events; a Happiness Index built from Andrews' single item of Happiness and the Life Satisfaction Scale. Adjusted multivariate analyses were performed. Results: Happiness was significantly associated with gender, marital status, poverty, and level of education. Parenting styles associated significantly with less happiness were: behaviors of rejection and sexist attitudes; and with higher happiness: emotional warmth. No association was found with overprotection or favoritism breeding practices. Early adverse events significantly associated with lower happiness were discussions within the family, father with problems of alcohol, parental absence, behaviors of emotional blackmail or threats. Conclusions: This study highlights the importance of developing intensive programs in the first years of life that promote parenting styles and healthy development environments.
\end{abstract}

Keywords: Happiness, Adult Survivors of Child Adverse Events, Child Rearing. (source: MeSH NLM)

\section{INTRODUCCIÓN}

Correspondencia: Javier E. Saavedra; jsaavedra@insm.gob.pe; javier;saavedra@upch.pe.

La felicidad corresponde al área de la psicología vinculada a la salud positiva. Las emociones como la felicidad, el contento o el disfrute amplían el repertorio de pensamiento-acción de las personas en un momento dado, por lo tanto, constituyen recursos personales más duraderos y servirían para prevenir y tratar problemas enraizados en emociones negativas, como la ansiedad, la depresión u otros relacionados con el estrés ${ }^{(1)}$. La salud positiva también se ha 
encontrado vinculada a correlatos biológicos como la sobrevivencia en personas con enfermedad cardíaca coronaria ${ }^{(2)}$, e inclusive con la longevidad ${ }^{(3)}$. Otros autores postulan que el crecimiento económico no necesariamente se correlaciona directamente con el sentimiento de felicidad, por lo que constituye una prioridad de población y una necesidad de investigación ${ }^{(4)}$.

El Reporte de Felicidad realizado por la Asamblea General de las Naciones Unidas, que utilizó dos preguntas para estimar la felicidad en los países ( «En general, teniendo todo en cuenta, ¿qué tan feliz se consideraría usted?»; y «Teniendo todo en cuenta, ¿qué tan satisfecho se encuentra usted con su vida como un todo en la actualidad?»), encontró que el Perú ocupaba el puesto 65 de 104 países, y el noveno en Latinoamérica, solo antes de Venezuela. Según este estudio los factores externos como determinantes de la felicidad fueron los ingresos, el trabajo, la satisfacción con la comunidad y la gobernanza, los valores y la religión; como factores personales, se señalaron la salud física, la salud mental, la experiencia de familia, la educación, el sexo y la edad ${ }^{(5)}$. Este estudio encontró que los países ricos tendían a ser más felices, aunque más importante para la felicidad que el ingreso eran los factores sociales como la fortaleza del soporte social, la ausencia de corrupción y el grado de libertad personal. Otros estudios han relacionado experiencias tempranas adversas y estilos de crianza con el bienestar subjetivo en la adultez, aunque menos con el sentimiento la felicidad ${ }^{(6,8)}$.

A nuestro entender no se han reportado otros estudios en la región, a nivel poblacional, que hayan explorado específicamente al sentimiento de felicidad y su relación con experiencias tempranas, por lo tanto, el objetivo del presente estudio ha sido estimar los factores sociodemográficos y los eventos de vida tempranos que se asocian con la felicidad en la población adulta de Lima Metropolitana.

\section{MATERIALES Y MÉTODOS}

\section{Diseño del estudio}

El presente estudio secundario de tipo transversal analítico empleó la base de datos del Estudio Epidemiológico de Salud Mental de Lima Metropolitana y Callao (EESMLMC), una encuesta realizada el 2012 y que utilizó una muestra representativa de personas adultas de Lima Metropolitana, seleccionada a través de un procedimiento probabilístico trietápico $^{(8)}$.

\section{Instrumentos}

Los instrumentos utilizados en el presente estudio han sido validados en estudios previos realizados por el Instituto Nacional de Salud Mental (INSM) en diversas regiones del Perú ${ }^{(9)}$ y fueron los siguientes:

Índice de calidad de vida de Mezzich: Es una escala de 10 ítems para evaluar aspectos relacionados al constructo de calidad de vida en una escala de 1 a 10.

\section{MENSAJES CLAVE}

Motivación para realizar el estudio: La salud pública ha colocado énfasis en estudiar aspectos negativos de la salud. Menos atención ha recibido el estudio de los factores que se asocian al bienestar de las personas y aspectos de salud positiva como la felicidad.

Principales hallazgos: La felicidad en el adulto se asoció con el sexo, estado civil, el grado de instrucción y el nivel de pobreza. Asimismo, la felicidad se asoció con los estilos de crianza recibidos y con eventos de vida tempranos.

Implicancias: Los eventos de vida tempranos tanto negativos como positivos pueden tener implicancias en el desarrollo de la felicidad de las personas.

La escala de propia memoria acerca de la crianza o EMBU (Egna Minen av Bardoms Uppfostran) versión resumida y modificada: En estudios previos del INSM, se elaboró una versión reducida de trece preguntas, a los que se agregaron otras cinco sobre favoritismo machista, autonomía personal, sobreprotección y control sin afecto ${ }^{(8)}$. El análisis psicométrico de estas 18 preguntas reportada por el INSM fue de una consistencia interna según el alfa de Cronbach de 0,74; se identificaron tres dimensiones: actitudes afectivas (de rechazo o calor), favoritismo con respecto a los hermanos, y sobreprotección y exigencias ${ }^{(9)}$.

Eventos vitales negativos antes de los 18 años. Se incluyeron 8 situaciones de eventos de vida adversos relativos a amenazas y condiciones de vida determinadas por otras conductas de los padres, considerados por el equipo de expertos de INSM como importantes en el contexto del país (Tabla 5).

Ítem único sobre felicidad de Andrews (IUFA) ${ }^{(10)}$ : Formulada con una sola pregunta con opciones de respuesta politómica de tipo categórica y ordenada; se obtiene un puntaje de 1 a 5 , donde 5 es el sentimiento de felicidad más alto: ¿se describiría a usted mismo como feliz e interesado por la vida? (5 puntos), ¿algo feliz? (4 puntos), ¿algo infeliz? (3 puntos), ¿infeliz y con poco interés en la vida? (2 puntos) y ¿tan infeliz que la vida no tiene sentido? (1 punto).

Escala de satisfacción con la vida de Diener (SVD) ${ }^{(11,12)}$ : Son 5 ítems en una escala de Likert de 5 puntos que miden la percepción de la satisfacción con la propia vida; la puntuación va de 5 a 25 y se considera 15 el puntaje neutral. Se obtienen finalmente 5 categorías: muy insatisfecho (5-9), insatisfecho (10-14), ni insatisfecho no satisfecho (15), satisfecho (16-20), y muy satisfecho (21-25). Para este estudio se reasignaron puntajes del 1 al 5 a las categorías según el nivel de satisfacción, donde un punto correspondió a muy insatisfecho y cinco puntos a muy satisfecho.

Índice de felicidad (IF): Se construyó este índice sumando los puntajes obtenidos del IUFA y los puntajes reasignados 
de SVD antes mencionados. Por lo tanto, la puntuación del IF fue de 2 a 10. Los puntos de corte se establecieron intencionalmente tomando primero los extremos, es decir, se consideraron personas con baja felicidad a aquellas personas que respondieron al IUFA como «algo infeliz» (3 puntos) y una respuesta de «ni satisfecho, ni insatisfecho» (3 puntos) en la SVD, lo que daba un puntaje de 6. Así, por un lado, se categorizó el IF como «baja felicidad» con un puntaje de 6 o menos e incluía a personas que se consideraban tan infelices que la vida no tenía sentido e insatisfechos con la vida. Por otro lado, se consideró como «alta o muy alta felicidad» si las respuestas a uno de los instrumentos tenían un puntaje máximo de 5 y el otro instrumento al menos un puntaje de 4 (9 o 10 puntos); y finalmente, se contempló como «mediana felicidad» (7 a 8 puntos) para el resto de combinaciones de respuestas con ambos instrumentos. Se programó para el estudio estimar la validez convergente del índice creado y sus categorías (material suplementario).

Datos demográficos e indicadores de pobreza: Se incluyeron preguntas de la Encuesta Nacional de Hogares (ENAHO) $2000^{(13)}$ sobre edad, sexo, estado civil, nivel educativo, situación laboral, área de residencia y sistema de salud adscrito, además de variables para el cálculo de la pobreza según la metodología de las necesidades básicas insatisfechas (NBI) como características de la vivienda, entre otros ${ }^{(14)}$. La presencia de dos o más NBI corresponde a familias en pobreza extrema, una NBI a familias pobres y la ausencia de NBI a familias no pobres.

\section{Análisis estadístico}

La muestra fue ponderada con el fin de reproducir la estructura demográfica de la población en estudio. Debido a que el IF fue creado para este estudio, se decidió evaluar la validez convergente estimando su asociación con el índice de calidad de vida, de Mezzich, siguiendo sugerencias teóricas alrededor de estos dos constructos ${ }^{(15)}$, así como el tipo de análisis estadístico requerido ${ }^{(16)}$. Como el IF era categórico y el índice de calidad de vida, numérico, se utilizó el análisis de varianza (ANOVA) que para el caso de muestras complejas implica, en el SPSS, el uso del modelo lineal general.

Para estimar la asociación entre el IF y las variables sociodemográficas e identificar las variables por ser incluidas en los modelos de regresión, se realizaron análisis bivariados con pruebas chi cuadrado convertidas al estadístico F como una variante del estadístico de chi cuadrado corregido, de Rao-Scott, de segundo orden y la significación basada en sus grados de libertad y un nivel de significancia de $\mathrm{p}<0,05$. Para el análisis multivariado se consideró el IF como variable respuesta y las variables sociodemográficas, calidad de vida, estilos de crianza y eventos de vida adversos como variables predictoras, cada una por separado. En cada caso se ajustó con las variables sociodemográficas identificadas al inicio con un nivel de significación de $\mathrm{p}<0,05$. Se aseguró que las variables predictoras cumplieran con el supuesto de no multicolinealidad. Se incluyeron análisis de regresión logística multinomial multivariada para estimar el OR ajustado y tener una mejor posibilidad de interpretación de la relación entre los factores estudiados y en cada uno de los niveles de felicidad.

\section{Consideraciones éticas}

El estudio original tuvo la aprobación del Comité de Ética del Instituto Nacional de Salud Mental «Honorio Delgado Hideyo Noguchi» y contó con la firma de los consentimientos informados de las personas entrevistadas. El presente estudio fue presentado al referido comité para exoneración de revisión por tratarse de un estudio que realiza un análisis secundario de una base de datos. La base datos utilizada para este estudio estuvo anonimizada.

\section{RESULTADOS}

Un total de 4445 adultos fueron entrevistados. La muestra ponderada fue distribuida en $48,0 \%$ varones y $52,0 \%$ mujeres. La edad promedio fue de 42,6 años (IC 95\%, 41,8-43,3). La mayoría de las personas encuestadas era casada o conviviente $(56,6 \%)$ y tenía instrucción secundaria $(44,8 \%)$; con una frecuencia de analfabetismo de 3,1\% (IC 95\%, 2,5-3,8); $63,7 \%$ (IC 95\%, 61,9-65,4) de la muestra había tenido un trabajo remunerado la semana anterior; y $21,5 \%$ de la población se encontraba en situación de pobreza según las NBI.

Según el IF, el 8,4\% (IC95\% 7,4-9,4) de la población expresaba baja o nula felicidad; el 29,9\% (IC 95\% 28,1-31,7) presentaba mediana felicidad; y el 61,7\% (IC 95\% 59,7-63,7) de la población manifestaba alta o muy alta felicidad. El tipo de felicidad reportada al momento de la encuesta según los factores sociodemográficos se muestran en la Tabla 1.

Los análisis bivariados mostraron una asociación significativa con el sexo, estado civil, nivel de instrucción, nivel de pobreza, lengua materna, analfabetismo y desocupación, con excepción de la edad. Sin embargo, en el análisis multivariado las variables lengua materna, analfabetismo y desocupación no continuaron mostrando diferencias significativas. Con respecto al sexo, se observa que los hombres tienen 1,79 veces más probabilidad de tener alta o muy alta felicidad, sobre baja felicidad, en comparación con las mujeres, el grupo que estuvo alguna vez unido (separado, divorciados o viudos) muestran una menor probabilidad de tener alta felicidad en comparación con las personas unidas (casadas o convivientes). Las personas con mayor instrucción tienen 3,18 veces más probabilidad que las personas de bajo nivel de instrucción de tener alta felicidad versus baja felicidad. Las personas no pobres tienen 2,54 veces más probabilidad que los pobres extremos de tener alta o muy alta felicidad versus la categoría de baja felicidad (Tabla 2). 
Tabla 1. Características sociodemográficas según niveles de felicidad autorreportada por adultos de Lima Metropolitana

\begin{tabular}{|c|c|c|c|c|c|}
\hline \multirow{2}{*}{ Característica } & \multirow[t]{2}{*}{$\mathbf{n}$} & \multirow[t]{2}{*}{$\%$} & \multirow{2}{*}{$\begin{array}{c}\text { Baja felicidad } \\
\% \text { (IC 95\%) }\end{array}$} & \multirow{2}{*}{$\begin{array}{c}\text { Mediana felicidad } \\
\% \text { (IC 95\%) }\end{array}$} & \multirow{2}{*}{$\begin{array}{c}\begin{array}{c}\text { Alta o muy alta } \\
\text { felicidad }\end{array} \\
\% \text { (IC 95\%) } \\
\end{array}$} \\
\hline & & & & & \\
\hline \multicolumn{6}{|l|}{ Sexo } \\
\hline Femenino & 2548 & 52,0 & $10,6(9,2-12,2)$ & $30,5(28,4-32,7)$ & $58,9(56,4-61,3)$ \\
\hline Masculino & 1897 & 48,0 & $5,9(4,8-7,2)$ & $29,3(26,7-31,9)$ & $64,8(62,0-67,5)$ \\
\hline \multicolumn{6}{|l|}{ Edad (años) } \\
\hline De 18 a 24 & 703 & 18,2 & $7,6(5,8-9,9)$ & $32,1(28,1-36,4)$ & $60,3(55,9-64,6)$ \\
\hline De 25 a 44 & 1970 & 40,6 & $9,0(7,6-10,6)$ & $28,0(25,5-30,6)$ & $63,0(60,2-65,8)$ \\
\hline De 45 a 64 & 1171 & 27,5 & $7,0(5,5-8,8)$ & $32,2(29,2-35,4)$ & $60,8(57,5-64,0)$ \\
\hline De 65 a más & 601 & 13,8 & $10,4(7,8-13,8)$ & $28,0(23,7-32,8)$ & $61,5(56,5-66,3)$ \\
\hline \multicolumn{6}{|l|}{ Estado civil } \\
\hline Unido & 2500 & 56,6 & $7,7(6,6-9,0)$ & $28,5(26,3-30,8)$ & $63,8(61,3-66,3)$ \\
\hline Alguna vez unido & 962 & 16,5 & $12,2(9,8-15,2)$ & $35,0(31,4-38,8)$ & $52,8(48,7-56,9)$ \\
\hline Nunca unido & 981 & 26,9 & $7,4(5,7-9,6)$ & $29,8(26,4-33,4)$ & $62,8(58,9-66,5)$ \\
\hline \multicolumn{6}{|l|}{ Nivel de instrucción } \\
\hline Sin nivel/Primaria & 632 & 18,3 & $12,5(9,8-15,7)$ & $38,5(34,2-43,0)$ & $49,0(44,5-53,5)$ \\
\hline Secundaria & 2037 & 44,8 & $10,4(8,9-12,2)$ & $33,3(30,7-36,0)$ & $56,3(53,5-59,1)$ \\
\hline Superior no universitaria & 851 & 20,0 & $5,0(3,6-6,9)$ & $27,3(23,8-31,2)$ & $67,6(63,6-71,4)$ \\
\hline Superior universitaria & 844 & 20,1 & $4,1(2,9-5,7)$ & $18,5(15,7-21,7)$ & $77,4(73,9-80,5)$ \\
\hline \multicolumn{6}{|l|}{ Nivel de pobreza } \\
\hline Pobre extremo & 248 & 4,4 & $15,3(10,9-21,0)$ & $44,0(37,5-50,7)$ & $40,7(33,5-48,4)$ \\
\hline Pobre & 853 & 17,1 & $11,0(8,7-13,8)$ & $37,7(33,7-41,9)$ & $51,3(46,9-55,6)$ \\
\hline No pobre & 3344 & 78,5 & $7,4(6,4-8,5)$ & $27,3(25,4-29,3)$ & $65,3(63,1-67,4)$ \\
\hline \multicolumn{6}{|l|}{ Lengua materna } \\
\hline Castellano & 3958 & 90,2 & $7,9(7,0-9,0)$ & $29,4(27,6-31,4)$ & $62,6(60,5-64,7)$ \\
\hline Quechua & 438 & 8,7 & $13,3(9,9-17,6)$ & $35,4(29,9-41,2)$ & $51,4(45,6-57,1)$ \\
\hline Aymara u otro & 46 & 11,0 & $8,6(3,0-22,5)$ & $23,8(12,5-40,6)$ & $67,6(48,5-82,3)$ \\
\hline \multicolumn{6}{|l|}{ Analfabetismo } \\
\hline Sí & 144 & 3,1 & $17,3(10,4-27,5)$ & $37,6(28,7-47,3)$ & $45,1(35,1-55,5)$ \\
\hline No & 4299 & 96,9 & $8,1(7,2-9,2)$ & $29,7(27,9-31,5)$ & $62,2(60,2-64,2)$ \\
\hline \multicolumn{6}{|l|}{ Desocupación } \\
\hline Sí & 1608 & 36,3 & $10,1(8,5-11,9)$ & $30,7(28,1-33,5)$ & $59,2(56,3-62,1)$ \\
\hline No & 2835 & 63,7 & $7,4(6,4-8,6)$ & $29,4(27,5-31,5)$ & $63,2(61,0-65,2)$ \\
\hline
\end{tabular}

Se encontró que existe una relación significativa entre las dimensiones del índice de calidad de vida y el IF según los puntajes que obtenían en el análisis bivariado y entre cada una de las categorías ajustado con variables sociodemográficas (Tabla 3).

Algunas de las variables de estilos de crianza están vinculadas de forma inversa a la felicidad, especialmente las relacionadas con el rechazo y las actitudes machistas, y en forma directa con estilos que implicaban calor emocional. Entre las que obtiene una asociación más intensa se encuentra: «Le dieron más castigos de los que merecía». Por otro lado, el estilo positivo más fuertemente asociado a la felicidad fue, "Existía amor y ternura entre usted y sus padres", donde las personas con estos antecedentes tuvieron 3,18 veces más probabilidad de tener alta o muy alta felicidad. No se asociaron estadísticamente con felicidad estilos de crianza que implicaban favoritismo o sobreprotección (Tabla 4).

Con respecto a eventos de vida adversos todas las situaciones consideradas mostraron una asociación significativa y una relación inversa intensa con la felicidad, resaltando situaciones como: «Alguno de sus padres lo(a) amenazaba con matarlo(a)» $\mathrm{y}$ «Alguno de sus padres lo(a) amenazaba con no quererlo(a)» (Tabla 5).

\section{DISCUSIÓN}

El presente estudio corresponde a uno de los primeros en el Perú que relaciona las variables expuestas. El estudio aporta evidencia sobre la importancia de la relación entre aspectos sociodemográficos y la felicidad, así como elementos de la historia personal que podrían influir en su futuro emocional. 
Tabla 2. Características sociodemográficas asociadas a la felicidad en adultos de Lima Metropolitana

\begin{tabular}{|c|c|c|c|c|}
\hline \multirow{3}{*}{ Factores sociodemográficos } & \multicolumn{2}{|c|}{ Análisis bivariado } & \multicolumn{2}{|c|}{ Análisis multivariado } \\
\hline & $\begin{array}{l}\text { Mediana vs. Baja } \\
\text { felicidad }\end{array}$ & $\begin{array}{c}\text { Alta o muy alta vs. Baja } \\
\text { felicidad }\end{array}$ & $\begin{array}{l}\text { Mediana vs. Baja } \\
\text { felicidad }\end{array}$ & $\begin{array}{c}\text { Alta o muy alta vs. Baja } \\
\text { felicidad }\end{array}$ \\
\hline & OR (IC 95\%) & OR (IC 95\%) & OR (IC 95\%) & OR (IC 95\%) ${ }^{a}$ \\
\hline \multicolumn{5}{|l|}{ Sexo } \\
\hline Femenino & 1,00 & 1,00 & 1,00 & 1,00 \\
\hline Masculino & $1,72(1,31-2,26)$ & $1,98(1,52-2,57)$ & $1,68(1,22-2,29)$ & $1,79(1,31-2,42)$ \\
\hline \multicolumn{5}{|l|}{ Edad (años) } \\
\hline De 65 a más & 1,00 & 1,00 & - & - \\
\hline De 45 a 64 & $1,72(1,10-2,69)$ & $1,48(0,98-2,24)$ & - & - \\
\hline De 25 a 44 & $1,15(0,76-1,75)$ & $1,19(0,81-1,73)$ & - & - \\
\hline De 18 a 24 & $1,58(0,99-2,51)$ & $1,35(0,87-2,10)$ & - & - \\
\hline \multicolumn{5}{|l|}{ Estado civil } \\
\hline Unido & 1,00 & 1,00 & 1,00 & 1,00 \\
\hline Alguna vez unido & $0,78(0,57-1,06)$ & $0,52(0,39-0,71)$ & $0,85(0,61-1,19)$ & $0,58(0,42-0,81)$ \\
\hline Nunca unido & $1,08(0,77-1,53)$ & $1,02(0,72-1,44)$ & $0,92(0,65-1,32)$ & $0,71(0,50-1,02)$ \\
\hline \multicolumn{5}{|l|}{ Nivel de instrucción } \\
\hline Sin nivel/Primaria & 1,00 & 1,00 & 1,00 & 1,00 \\
\hline Secundaria & $1,04(0,73-1,48)$ & $1,38(0,99-1,92)$ & $0,80(0,53-1,18)$ & $1,02(0,70-1,50)$ \\
\hline Superior no universitaria & $1,76(1,12-2,79)$ & $3,43(2,20-5,34)$ & $1,40(0,84-2,32)$ & $2,54(1,54-4,18)$ \\
\hline Superior universitaria & $1,47(0,91-2,36)$ & $4,82(3,05-7,60)$ & $1,08(0,64-1,82)$ & $3,18(1,90-5,31)$ \\
\hline \multicolumn{5}{|l|}{ Nivel de pobreza } \\
\hline Pobre extremo & 1,00 & 1,00 & 1,00 & 1,00 \\
\hline Pobre & $1,19(0,74-1,91)$ & $1,75(1,04-2,94)$ & $1,11(0,68-1,82)$ & $1,58(0,93-2,68)$ \\
\hline No pobre & $1,29(0,86-2,94)$ & $3,33(2,08-5,33)$ & $1,15(0,75-1,78)$ & $2,54(1,58-4,11)$ \\
\hline \multicolumn{5}{|l|}{ Lengua materna } \\
\hline Aymara u otro & 1,00 & 1,00 & 1,00 & 1,00 \\
\hline Quechua & $0,96(0,29-3,25)$ & $0,49(0,14-1,74)$ & $0,98(0,27-3,63)$ & $0,55(0,12-2,54)$ \\
\hline Castellano & $1,34(0,43-4,25)$ & $1,01(0,30-3,37)$ & $1,25(0,36-4,32)$ & $0,78(0,18-3,42)$ \\
\hline \multicolumn{5}{|l|}{ Analfabetismo } \\
\hline Sí & 1,00 & 1,00 & 1,00 & 1,00 \\
\hline No & $1,69(0,89-3,21)$ & $2,95(1,52-5,70)$ & $1,35(0,65-2,78)$ & $1,27(0,59-2,70)$ \\
\hline \multicolumn{5}{|l|}{ Desocupación } \\
\hline Sí & 1,00 & 1,00 & 1,00 & 1,00 \\
\hline No & $1,30(0,99-1,70)$ & $1,44(1,11-1,87)$ & $1,12(0,82-1,52)$ & $1,14(0,85-1,53)$ \\
\hline
\end{tabular}

ajustado por sexo, estado civil, nivel de instrucción y pobreza, analfabetismo lengua materna y desocupación

Una gran proporción de la población adulta de Lima considera experimentar un alto o muy alto nivel de felicidad. Estos hallazgos son semejantes con la Encuesta sobre Felicidad, Esperanza y Optimismo Económico, realizado por Gallup Internacional, que encontró que el 59\% de la población mundial refería sentirse feliz, o muy feliz y en el caso de Latinoamérica, el $69 \%{ }^{(17)}$. Con respecto a las variables sociodemográficas, este estudio encontró una asociación significativa con sexo, estado civil, nivel de instrucción y pobreza. Estos hallazgos son compatibles con otro estudio realizado en Irán ${ }^{(18)}$. Otras variables como analfabetismo, lengua materna y desocupación si bien estuvieron significativamente asociadas con la felicidad en los análisis bivariados, no conservaron la asociación al momento de controlar con otras variables sociodemográficas. Asimismo, algunos estudios no han encontrado asociación entre sexo y felicidad ${ }^{(19)}$.

Con respecto a la relación entre pobreza y felicidad, algunos autores postulan que la percepción de las diferencias sociales con las demás personas lleva a la búsqueda de satisfacer las necesidades materiales o físicas que inciden en un 
Tabla 3. Calidad de vida asociado a felicidad en adultos de Lima Metropolitana

\begin{tabular}{|c|c|c|c|c|c|c|c|}
\hline \multirow[b]{3}{*}{ Índice de calidad de vida } & & & \multicolumn{3}{|c|}{ Análisis bivariado $^{\mathrm{a}}$} & \multicolumn{2}{|c|}{ Análisis multivariado $^{\mathrm{b}}$} \\
\hline & & & Baja felicidad & $\begin{array}{l}\text { Mediana } \\
\text { felicidad }\end{array}$ & $\begin{array}{c}\text { Alta o muy alta } \\
\text { felicidad } \\
\end{array}$ & $\begin{array}{c}\text { Mediana vs. baja } \\
\text { felicidad }\end{array}$ & $\begin{array}{r}\text { Alta o muy alta } \\
\text { vs. baja felicidad }\end{array}$ \\
\hline & $\mathbf{n}$ & $\begin{array}{c}\text { Promedio } \\
\text { poblacional (EE) }\end{array}$ & $\begin{array}{c}\begin{array}{c}\text { Promedio } \\
\text { (IC 95\%) }\end{array} \\
\end{array}$ & $\begin{array}{c}\text { Promedio } \\
\text { (IC 95\%) }\end{array}$ & $\begin{array}{c}\text { Promedio } \\
\text { (IC 95\%) }\end{array}$ & OR (IC 95\%) $)^{c}$ & OR $(\operatorname{IC~} 95 \%)^{c}$ \\
\hline \multicolumn{8}{|l|}{ Dimensiones } \\
\hline Bienestar físico & 4413 & $7,59(0,04)$ & $6,32(6,13-6,52)$ & $7,13(7,03-7,24)$ & $7,97(7,89-8,06)$ & $1,32(1,22-1,43)$ & $1,87(1,72-2,03)$ \\
\hline $\begin{array}{l}\text { Bienestar psicológico o } \\
\text { emocional }\end{array}$ & 4413 & $7,98(0,03)$ & $6,45(6,24-6,66)$ & $7,59(7,49-7,68)$ & $8,38(8,31-8,45)$ & $1,53(1,4-1,67)$ & $2,27(2,07-2,49)$ \\
\hline $\begin{array}{l}\text { Autocuidado } \\
\text { y funcionamiento }\end{array}$ & 4414 & $8,50(0,03)$ & $7,70(7,51-7,89)$ & $8,25)(8,15-8,34)$ & $8,74(8,68-8,80)$ & $1,28(1,17-1,40)$ & $1,67(1,51-1,83)$ \\
\hline $\begin{array}{l}\text { Funcionamiento } \\
\text { ocupacional }\end{array}$ & 4412 & $8,47(0,03)$ & $7,66(7,46-7,87)$ & $8,20(8,10-8,30)$ & $8,70(8,64-8,77)$ & $1,24(1,14-1,34)$ & $1,58(1,45-1,73)$ \\
\hline $\begin{array}{l}\text { Funcionamiento } \\
\text { interpersonal }\end{array}$ & 4411 & $8,30(0,03)$ & $7,33(7,12-7,54)$ & $7,96(7,86-8,06)$ & $8,59(8,53-8,65)$ & $1,28(1,17-1,40)$ & $1,76(1,60-1,92)$ \\
\hline Apoyo social-emocional & 4410 & $7,77(0,04)$ & $6,67(6,45-6,89)$ & $7,35(7,23-7,48)$ & $8,11(8,03-8,20)$ & $1,18(1,11-1,26)$ & $1,50(1,40-1,61)$ \\
\hline $\begin{array}{l}\text { Apoyo comunitario y de } \\
\text { servicios }\end{array}$ & 4386 & $6,43(0,05)$ & $5,39(5,14-5,65)$ & $6,22(6,07-6,37)$ & $6,68(6,57-6,79)$ & $1,18(1,11-1,24)$ & $1,31(1,24-1,38)$ \\
\hline Plenitud personal & 4412 & $7,81(0,04)$ & $6,39(6,17-6,61)$ & $7,34(7,24-7,45)$ & $8,23(8,16-8,30)$ & $1,37(1,26-1,48)$ & $1,99(1,82-2,17)$ \\
\hline Satisfacción espiritual & 4409 & $8,13(0,03)$ & $7,10(6,90-7,30)$ & $7,76(7,66-7,86)$ & $8,44(8,37-8,51)$ & $1,27(1,17-1,36)$ & $1,73(1,59-1,89)$ \\
\hline Calidad de vida global & 4414 & $8,24(0,03)$ & $6,82(6,64-7,01)$ & $7,79(7,69-7,88)$ & $8,64(8,58-8,71)$ & $1,46(1,35-1,59)$ & $2,38(2,17-2,62)$ \\
\hline Puntaje total ICV & 4416 & $7,92(0,03)$ & $6,78(6,65-6,92)$ & $7,56(7,49-7,63)$ & $8,25(8,20-8,30)$ & $1,93(1,69-2,20)$ & $3,71(3,22-4,28)$ \\
\hline
\end{tabular}

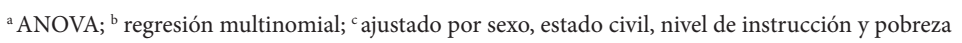

EE: error estándar

detrimento del tiempo dedicado para las relaciones sociales, que son las que se consideran que generan felicidad en las personas ${ }^{(4)}$. Sin embrago, debemos resaltar que una proporción importante de personas pobres extremas estuvieron en el nivel de alta o muy alta felicidad. Algunos autores resaltan que la situación de pobreza puede favorecer el colectivismo o la propensión a integrarse en grupos y explicaría por qué algunos países pobres tendrían una percepción de felicidad más alta que algunos países ricos ${ }^{(20)}$.

Con relación a la calidad de vida y la felicidad, este estudio se propuso analizar la relación entre ambas variables como una forma de validar el IF utilizado como instrumento de medición de la felicidad y encontró una asociación significativa entre todas las dimensiones del índice de calidad de vida y los niveles de felicidad y corroboró propuestas de otros autores sobre la correlación entre ambos ${ }^{(15)}$. Esta relación también se expresa en los hallazgos que indicarían que la felicidad está asociada a diversos resultados de éxito en el trabajo, ingresos, involucramiento comunitario, las relaciones interpersonales, y la salud en general, todos relacionados con el concepto de calidad de vida ${ }^{(21)}$.

Con respecto a los estilos de crianza, los eventos adversos tempranos no solo se asocian a problemas de trastornos mentales en la adultez ${ }^{(22)}$, sino que también se asocian a aspectos de salud positiva, como la felicidad. Un estudio de 3292 personas, reportado en Japón, encontró que las experiencias adversas en la niñez se relacionaba con el bienestar subjetivo en la adultez, independientemente de los factores socioeconómicos ${ }^{(7)}$. Por el contrario, el estudio desarrollado por Caycho y colaboradores con estudiantes universitarios en Lima ${ }^{(6)}$ no encontró relación significativa entre felicidad y los estilos de crianza de sobreprotección y favoritismo. Una explicación puede estar en la edad y el contexto socioeconómico de los participantes, ya que el estudio de Caycho se realizó en adultos jóvenes de universidades privadas. Sin embargo, no puede descartarse que la relación entre eventos adversos en la niñez y felicidad este mediado por otros factores. En este sentido, un estudio reportó que la autoestima es un moderador importante entre los estilos parentales y la felicidad y que el estilo democrático de la madre, vinculado a la autoestima, era el más asociado con la felicidad.

Se encontró una relación negativa entre eventos de vida adversos a la felicidad. Estos hallazgos son coherentes con un estudio en la comunidad que ha reportado una relación entre la intensidad del maltrato en la infancia y el estado de salud mental en la adultez ${ }^{(23)}$. Otros autores han encontrado que el maltrato en la infancia se correlaciona negativamente con diversos indicadores de bienestar, autoestima, felicidad y satisfacción con la vida en el adulto ${ }^{(24)}$. Una explicación a esta relación puede ser que experiencias traumáticas tempranas se han relacionado con la desregulación emocional ${ }^{(25)}$, y estudios de seguimiento de desregulación emocional en la infancia 14 años después han reportado un mayor incremento de problemas emocionales en la adultez ${ }^{(26)}$. Un estudio en 17337 adultos de atención primaria demostró un efecto acumulativo de las experiencias adversas en la infancia en diversos dominios emocionales y conductuales en la adultez, lo que sería una posible explicación de una menor felicidad de las personas con historias de eventos adversos en su infancia ${ }^{(27)}$.

Dentro de la felicidad, se ha considerado desarrollar la tolerancia ${ }^{(28)}$, nutrir la espiritualidad, dar prioridad a las 
Tabla 4. Estilos de crianza asociados a felicidad en adultos de Lima Metropolitana

\begin{tabular}{|c|c|c|c|c|c|c|}
\hline \multirow[b]{3}{*}{ Estilos de crianza } & \multirow[b]{3}{*}{$\mathbf{n}$} & \multirow[b]{3}{*}{$\%$} & \multicolumn{2}{|c|}{ Análisis bivariado } & \multicolumn{2}{|c|}{ Análisis multivariado } \\
\hline & & & $\begin{array}{l}\text { Mediana vs. baja } \\
\text { felicidad }\end{array}$ & $\begin{array}{l}\text { Alta o muy alta } \\
\text { vs. baja felicidad }\end{array}$ & $\begin{array}{l}\text { Mediana vs. baja } \\
\text { felicidad }\end{array}$ & $\begin{array}{l}\text { Alta o muy alta } \\
\text { vs. baja felicidad }\end{array}$ \\
\hline & & & OR (IC 95\%) & OR (IC 95\%) & OR (IC 95\%) ${ }^{\mathrm{a}}$ & OR (IC 95\%) $)^{\mathrm{a}}$ \\
\hline \multicolumn{7}{|l|}{ Estilo afectivo negativo (control y rechazo) } \\
\hline Le dieron más castigos de los que merecía & 969 & 20,0 & $0,60(0,45-0,78)$ & $0,30(0,23-0,40)$ & $0,62(0,47-0,82)$ & $0,34(0,26-0,45)$ \\
\hline $\begin{array}{l}\text { Lo/la castigaban severamente (o mucho) por } \\
\text { cosas pequeñas }\end{array}$ & 726 & 14,8 & $0,76(0,57-1,01)$ & $0,40(0,30-0,54)$ & $0,80(0,60-1,07)$ & $0,46(0,34-0,62)$ \\
\hline Lo/la controlaban, pero no le daban afecto & 496 & 9,9 & $0,79(0,55-1,15)$ & $0,42(0,30-0,60)$ & $0,84(0,58-1,22)$ & $0,47(0,33-0,67)$ \\
\hline \multicolumn{7}{|l|}{ Actitudes machistas } \\
\hline $\begin{array}{l}\text { Sus padres pensaban que los hijos hombres no } \\
\text { deberían llorar }\end{array}$ & 1083 & 23,9 & $0,90(0,67-1,22)$ & $0,62(0,47-0,83)$ & $0,92(0,68-1,25)$ & $0,69(0,51-0,93)$ \\
\hline $\begin{array}{l}\text { Sus padres preferían a los hijos varones en } \\
\text { comparación con las hijas mujeres }\end{array}$ & 599 & 12,6 & $0,68(0,49-0,94)$ & $0,39(0,28-0,54)$ & $0,74(0,53-1,03)$ & $0,47(0,34-0,66)$ \\
\hline \multicolumn{7}{|l|}{ Estilo afectivo positivo (calor emocional) } \\
\hline $\begin{array}{l}\text { Si las cosas le iban mal, sus padres trataban de } \\
\text { confortarlo(a), animarlo(a) y apoyarlo(a) }\end{array}$ & 3397 & 79,2 & $1,46(1,10-1,92)$ & $3,25(2,50-4,22)$ & $1,38(1,04-1,83)$ & $2,88(2,20-3,76)$ \\
\hline Existía amor y ternura entre usted y sus padres & 3842 & 88,9 & $1,41(1,03-1,95)$ & $3,80(2,79-5,19)$ & $1,3(0,93-1,82)$ & $3,18(2,30-4,40)$ \\
\hline Le demostraban que lo/laquerían & 3866 & 88,8 & $1,19(0,85-1,64)$ & $2,41(1,74-3,33)$ & $1,12(0,80-1,56)$ & $2,16(1,56-2,99)$ \\
\hline $\begin{array}{l}\text { Le permitían tomar sus propias decisiones } \\
\text {, por ejemplo, elegir su ropa, escoger a sus } \\
\text { amistades, los estudios que debía seguir, } \\
\text { distracciones, etc. }\end{array}$ & 2058 & 49,1 & $1,18(0,89-1,58)$ & $1,77(1,35-2,32)$ & $1,09(0,81-1,47)$ & $1,51(1,13-2,01)$ \\
\hline $\begin{array}{l}\text { Le demostraban que estaban interesados en } \\
\text { que usted tuviera buenas calificaciones }\end{array}$ & 3189 & 74,7 & $1,57(1,21-2,04)$ & $2,59(1,99-3,37)$ & $1,48(1,13-1,94)$ & $2,17(1,65-2,86)$ \\
\hline $\begin{array}{l}\text { Sus padres se interesaban por conocer sus } \\
\text { opiniones (o ideas) }\end{array}$ & 2720 & 63,7 & $1,54(1,17-2,03)$ & $2,92(2,23-3,81)$ & $1,46(1,09-1,95)$ & $2,54(1,91-3,37)$ \\
\hline \multicolumn{7}{|l|}{ Favoritismo } \\
\hline $\begin{array}{l}\text { Le permitían hacer cosas que a sus } \\
\text { hermanos(as) no les era permitido }\end{array}$ & 556 & 12,8 & $1,07(0,75-1,55)$ & $0,91(0,64-1,29)$ & $1,04(0,72-1,5)$ & $0,87(0,60-1,25)$ \\
\hline $\begin{array}{l}\text { Lo/la engreían más a usted en comparación } \\
\text { con sus hermanos(as) }\end{array}$ & 690 & 16,1 & $1,12(0,76-1,64)$ & $1,09(0,75-1,59)$ & $1,16(0,79-1,71)$ & $1,19(0,81-1,73)$ \\
\hline $\begin{array}{l}\text { Lo/la favorecían con relación a sus } \\
\text { hermanos(as) }\end{array}$ & 612 & 13,9 & $1,27(0,87-1,85)$ & $1,2(0,83-1,72)$ & $1,33(0,9-1,95)$ & $1,34(0,92-1,96)$ \\
\hline \multicolumn{7}{|l|}{ Sobreprotección } \\
\hline $\begin{array}{l}\text { Le prohibían hacer cosas que a otros niños(as) } \\
\text { sí les era permitido, porque temían que algo } \\
\text { malo le podría ocurrir }\end{array}$ & 1500 & 33,0 & $1,04(0,80-1,36)$ & $1,15(0,89-1,47)$ & $1,02(0,78-1,34)$ & $1,08(0,83-1,39)$ \\
\hline $\begin{array}{l}\text { Eran muy exigentes con respecto a sus } \\
\text { calificaciones escolares, desempeño deportivo } \\
\text { o actividades similares }\end{array}$ & 2058 & 45,0 & $1,22(0,93-1,61)$ & $1,36(1,06-1,75)$ & $1,18(0,89-1,55)$ & $1,26(0,97-1,64)$ \\
\hline $\begin{array}{l}\text { La ansiedad de sus padres de que algo malo } \\
\text { podía sucederle era exagerada }\end{array}$ & 987 & 22,2 & $1,1(0,81-1,49)$ & $1,05(0,78-1,41)$ & $1,11(0,82-1,50)$ & $1,08(0,80-1,46)$ \\
\hline $\begin{array}{l}\text { Sus padres lo(a) cuidaban excesivamente } \\
\text { (o demasiado) para evitar que tenga problemas }\end{array}$ & 1134 & 25,1 & $1,15(0,86-1,53)$ & $0,99(0,75-1,31)$ & $1,18(0,89-1,56)$ & $1,07(0,81-1,41)$ \\
\hline
\end{tabular}

a Ajustado por sexo, estado civil, nivel de instrucción y pobreza

relaciones cercanas, actuar positivamente frente a las circunstancias, controlar las emociones estresantes, procurar un ambiente físico agradable, promover actividades físicas saludables, buscar trabajos que sean compatibles con sus habilidades, incluir la recreación en la vida, no hacer comparaciones y preocuparse por otras personas ${ }^{(29)}$. Poca atención se le da en la atención primaria a la identificación de experiencias adversas en la niñez, a pesar del impacto que tiene ${ }^{(30)}$. Considerar la felicidad en el centro de las políticas de los gobiernos viene sugerido por el hecho que el crecimiento económico en los países desarrollados no se ha acompañado de una mejoría en las encuestas sobre felici- 
Tabla 5. Eventos de vida adversos asociados a felicidad en adultos de Lima Metropolitana

\begin{tabular}{|c|c|c|c|c|c|c|}
\hline \multirow[b]{3}{*}{ Estilos de crianza } & & & \multicolumn{2}{|c|}{ Análisis bivariado } & \multicolumn{2}{|c|}{ Análisis multivariado } \\
\hline & & & $\begin{array}{l}\text { Mediana vs. baja } \\
\text { felicidad } \\
\end{array}$ & $\begin{array}{c}\text { Alta o muy alta } \\
\text { vs. baja felicidad } \\
\end{array}$ & $\begin{array}{c}\text { Mediana vs. baja } \\
\text { felicidad }\end{array}$ & $\begin{array}{l}\text { Alta o muy alta } \\
\text { vs. baja felicidad }\end{array}$ \\
\hline & $\mathbf{n}$ & $\%$ & OR (IC 95\%) & OR (IC 95\%) & OR (IC 95\%) $)^{\mathrm{a}}$ & OR (IC 95\%) ${ }^{a}$ \\
\hline $\begin{array}{l}\text { Cuando era menor de } 11 \text { años sus padres discutían } \\
\text { mucho }\end{array}$ & 1325 & 29,9 & $0,87(0,66-1,16)$ & $0,55(0,42-0,72)$ & $0,89(0,67-1,18)$ & $0,59(0,45-0,78)$ \\
\hline $\begin{array}{l}\text { Alguno de sus padres llegaba ebrio o borracho a } \\
\text { casa con regularidad (o frecuencia) }\end{array}$ & 1323 & 28,3 & $0,86(0,66-1,13)$ & $0,52(0,41-0,67)$ & $0,89(0,68-1,17)$ & $0,59(0,45-0,76)$ \\
\hline $\begin{array}{l}\text { Alguno de sus padres estaba mucho tiempo ausen- } \\
\text { te de casa }\end{array}$ & 1247 & 27,1 & $0,71(0,54-0,92)$ & $0,45(0,35-0,58)$ & $0,72(0,55-0,94)$ & $0,47(0,37-0,61)$ \\
\hline Alguno de sus padres fue infiel en la relación & 916 & 20,2 & $0,78(0,58-1,05)$ & $0,53(0,40-0,71)$ & $0,78(0,58-1,06)$ & $0,53(0,40-0,71)$ \\
\hline $\begin{array}{l}\text { Alguno de sus padres lo(a) amenazaba con no que- } \\
\text { rerlo(a) }\end{array}$ & 164 & 3,2 & $0,53(0,31-0,89)$ & $0,22(0,13-0,38)$ & $0,55(0,33-0,93)$ & $0,27(0,15-0,47)$ \\
\hline $\begin{array}{l}\text { Alguno de sus padres lo(a) amenazaba con matar- } \\
\text { lo(a) }\end{array}$ & 43 & 0,9 & $0,63(0,25-1,58)$ & $0,14(0,05-0,38)$ & $0,64(0,24-1,65)$ & $0,16(0,06-0,44)$ \\
\hline $\begin{array}{l}\text { Alguno de sus padres lo(a) hacía sentir culpable } \\
\text { por desacuerdos o discusiones de la familia }\end{array}$ & 227 & 4,5 & $0,6(0,38-0,95)$ & $0,30(0,19-0,48)$ & $0,62(0,39-1,00)$ & $0,33(0,21-0,53)$ \\
\hline $\begin{array}{l}\text { Alguno de sus padres lo(a) amenazaba con aban- } \\
\text { donarlo(a) }\end{array}$ & 150 & 3,1 & $0,49(0,29-0,82)$ & $0,21(0,13-0,35)$ & $0,51(0,30-0,87)$ & $0,25(0,15-0,42)$ \\
\hline
\end{tabular}

a Ajustado por sexo, estado civil, nivel de instrucción y pobreza

dad. Sin embargo, aún persisten controversias relacionadas a la alta subjetividad ${ }^{(31)}$.

Los resultados de este estudio deben ser considerados con las siguientes limitaciones. El indicador de felicidad utilizado ha sido creado para el estudio $y$, a pesar de tener evidencia de su validez convergente, podrían ser necesarios otros estudios de validación. Este estudio ha explorado solo algunos aspectos de eventos tempranos y algunos estilos de crianza y no ha integrado todos los factores que pueden mediar y ser importantes para la felicidad. Asimismo, no podemos descartar que el efecto de la memoria y de la deseabilidad social haya influenciado en las respuestas de los entrevistados.

En conclusión, los hallazgos muestran la importancia de los eventos de vida tempranos tanto negativos como positivos para el desarrollo de la felicidad de las personas. Asimismo, contribuye con información relevante para un cambio de paradigma en el estudio de la salud mental con un enfo- que de salud pública no centrado en las enfermedades sino en la salud en su totalidad. El presente estudio agrega evidencia sobre la importancia de desarrollar programas dirigidos a los primeros años de vida de las personas, con medidas promocionales y preventivas en el desarrollo de conductas saludables de crianza y entornos propicios para el bienestar en la vida de las personas.

Contribuciones del autor: JES ha participado en la concepción de la investigación, diseño del artículo, análisis de datos, redacción y aprobación de la versión final.

Fuentes de financiamiento: El estudio original se realizó con financiamiento del Instituto Nacional de Salud Mental.

Conflictos de interés: El autor no declara ningún conflicto de interés.

Material suplementario: Disponible en la versión electrónica de la RPMESP

\section{REFERENCIAS BIBLIOGRÁFICAS}

1. Fredrickson BL, Tugade MM, Waugh CE, Larkin GR. What good are positive emotions in crises? a prospective study of resilience and emotions following the terrorist attacks on the united states on september 11th, 2001. J Pers Soc Psychol. 2003; 84(2): 365-376.

2. Hoen PW, Denollet J, de Jonge P, Whooley MA. Positive affect and survival in patients with stable coronary heart disease: findings from the Heart and Soul Study. J Clin Psychiatry. 2013;74(7):716-22.

3. Koopmans TA, Geleijnse JM, Zitman FG, Giltay EJ. Effects of Happiness on all-cause mortality during 15 years of follow-up: The Arnhem Elderly Study. J Happiness Stud. 2010; 11(1):113-124.

4. Stillwell F, Jordan K. Economic Inequality and (Un)happiness. Social Alternatives. 2007; 26(4):16-21.

5. Helliwell JF, Layard R, Sachs JD. World Happiness Report 2018. New York: Sustainable Development Solutions Network; 2018. Disponible en: https://s3.amazonaws.com/happiness-report/2018/WHR_web.pdf.
6. Caycho Rodríguez T, Contreras Paredes K, Merino Soto C. Percepción de los estilos de crianza y felicidad en adolescentes y jóvenes de Lima Metropolitana. Perspect Fam. 2016; (1):11-22.

7. Oshio T, Umeda M, Kawakami N. Childhood Adversity and Adulthood Subjective Well-Being: Evidence from Japan. J Happiness Stud. 2013; 14:843-860. doi 10.1007/s10902-012-9358-y.

8. Instituto Nacional de Salud Mental. Estudio Epidemiológico de Salud Mental en Lima Metropolitana y Callao - Replicación 2012 - Informe General. Anales de Salud Mental. 2013; 29 (Suplemento 1): 1-392.

9. Instituto Nacional de Salud Mental. Confiabilidad y validez de los cuestionarios de salud mental de Lima y de la Selva Peruana. Anales de Salud Mental 2009; XXV (Supl. 1): 1-259.

10. Andrews FM. Social Indicators of Perceived Life Quality. Social Indicators Research. 1974; 1: 279-299.

11. Pavot W, Diener E. Review of the Satisfaction with life scale. Psychological Assessment 1993; 5 (2):164-172. doi 10.1007/978-90-481-2354-4 5 
12. Ly G. Atribuciones causales de la satisfacción con la vida en un grupo de adultos de Lima [Tesis de Licenciatura en Psicología]. Lima: Pontificia Universidad Católica del Perú; 2004.

13. INEI. Manual de la Encuestadora de la Encuesta Nacional de Hogares 2000 IV trimestre Oct 2000. Lima: INEI; 2000.

14. Feres JC, Macero X. Enfoques para la medición de la pobreza. Breve revisión de la literatura. Santiago de Chile: UN; 2001. [Citado el 3 de noviembre de 2005] Disponible en: http://www.eclac.org/publicaciones/ Estadisticas/9/lcl1479/ lcl1479e.pdf.

15. Susniene D, Jurkauskas A. The Concepts of Quality of Life and Happiness - Correlation and Differences. Inzinerine Ekonomika-Engineering Economics. 2009; 3: 48-55.

16. Loewenthal KM. The final scale and its validation. En: Autor. An introduction to psychological tests and scales. London: UCL Press Limited; 1996. p. 55-60.

17. Gallup International. Happiness, Hope, Economic Optimism.[Internet]. Washington DC: Gallup International; 2017 [citado el 31 de mayo del 2019]. Disponible en: http://www.gallup-international. com/wp-content/uploads/2017/12/2017_Happiness_Hope_Economic-Optimism.pdf

18. Azizi M, Mohamadian F, Ghajarieah M, Direkvand-Moghadam A. The Effect of Individual Factors, Socioeconomic and Social Participation on Individual Happiness: A Cross-Sectional Study. J Clin Diagn Res. 2017;11(6):VC01-VC04. doi: 10.7860/JCDR/2017/24658.9982

19. Mukherjee D, Basu S. A Study of Contributory Factors of Happiness. Indian Journal of Positive Psychology. 2012; 3(2): 136-142.

20. Borrero S, Bolena Escobar A, Cortes AM, Maya LC. Poor and distressed, but happy: situational and cultural moderators of the relationship between wealth and happiness. Estudios Gerenciales [Internet]. 2013 [citado el 31 de mayo del 2019]; 29 (126):2-11. Disponible en: http://www.scielo.org. co/pdf/eg/v29n126/v29n126a02.pdf

21. Lyubomirsky S, King L, Diener E. The Benefits of Frequent Positive Affect: Does Happiness Lead to Success?. Psychological Bulletin. 2005; 131(6):803-855.
22. Green JG, McLaughlin KA, Berglund PA, Gruber MJ, Sampson NA, Zaslavsky AM, Kessler RC. Childhood adversities and adult psychiatric disorders in the national comorbidity survey replication I: associations with first onset of DSM-IV disorders. Arch Gen Psychiatry. 2010;67(2):113-23. doi: 10.1001/archgenpsychiatry.2009.186.

23. Edwards VJ, Holden GW, Felitti VJ, Anda RF. Relationship between multiple forms of childhood maltreatment and adult mental health in community respondents: results from the adverse childhood experiences study. Am J Psychiatry. 2003;160(8):1453-60.

24. Herrenkohl TI, Klika JB, Herrenkohl RC, Russo MJ, Dee T. A prospective investigation of the relationship between child maltreatment and indicators of adult psychological well-being. Violence Vict. 2012;27(5):764-76.

25. Kim J, Cicchetti D. Longitudinal pathways linking child maltreatment, emotion regulation, peer relations, and psychopathology. J Child Psychol Psychiatry. 2010;51(6):706-16. doi: 10.1111/j.1469-7610.2009.02202.x

26. Althoff RR, Verhulst FC, Rettew DC, Hudziak JJ, van der Ende J. Adult outcomes of childhood dysregulation: a 14-year follow-up study. J Am Acad Child Adolesc Psychiatry. 2010;49(11):1105-16. doi: 10.1016/j. jaac.2010.08.006.

27. Anda RF, Felitti VJ, Bremner JD, Walker J, Whitfield C, Perry BD et al. The enduring effects of abuse and related adverse experiences in childhood: A convergence of evidence from neurobiology and epidemiology. Eur Arch Psychiatry Clin Neurosci. 2006;256(3):174-186.

28. Inglehart RF, Borinskaya S, Cotter A, Harro J, Inglehart RC, Ponarin E, Welzel C. Genetic Factors, Cultural Predispositions, Happiness and Gender Equality. Journal of Research in Gender Studies. 2014; 4(1): 32-100.

29. Malik A, Singh D. Happiness: A central objective of positive psychology. Indian Journal of Positive Psychology. 2012; 3(1):101-104.

30. Weinreb L, Savageau JA, Candib LM, Reed GW, Fletcher KE, Hargraves JL. Screening for childhood trauma in adult primary care patients: a cross-sectional survey. Prim Care Companion J Clin Psychiatry. 2010;12(6). pii: PCC.10m00950 doi: 10.4088/PCC.10m00950blu

31. Duncan G. Should happiness-maximization be the goal of government? J Happiness Stud. 2010; 11:163-178. 